TUM-T31-80/94

(hep-ph/9410288)

October 1994

\title{
Studying Structure of Electroweak Corrections
}

\author{
ZENRŌ HIOKIPE' \\ Physik Department, Technische Universität München \\ D-85748 Garching, F. R. GERMANY
}

\begin{abstract}
Several different effects in electroweak quantum corrections are explored separately through the latest data on the weak-boson masses. The leading-log approximation, the improved-Born approximation and the non-decoupling top-quark effects are studied without depending on the recent CDF data of $m_{t}$, and the results are given in a form independent of the Higgs mass. On the other hand, the bosonic- and the non-decoupling Higgs effects are examined by fully taking account of those CDF data. It is emphasized that future precision measurements of $M_{W}$ and $m_{t}$ are considerably significant not only for further studies of the electroweak theory at higher confidence level but also for new physics searches beyond it.
\end{abstract}

\footnotetext{
1) Fellow of the Alexander von Humboldt Foundation

2) Address after Nov. 1, 1994: Institute of Theoretical Physics, University of Tokushima, Tokushima 770, JAPAN (E-mail: hioki@ias.tokushima-u.ac.jp)
} 


\section{$\S 1$. Introduction}

Analyses of electroweak radiative corrections have been extensively carried out since high-energy experiments at $O\left(10^{2}\right) \mathrm{GeV}$ scale started at SLAC, FNAL and CERN. In particular, a lot of data on $Z$ from LEP collaborations at CERN have enabled us to test the minimal standard electroweak theory (hereafter simply "electroweak theory") with considerable accuracy [1]-[4] (see also [5] as the latest review articles). Furthermore, CDF collaboration at FNAL Tevatron collider reported recently some evidence on top-quark pair productions and estimated thereby its mass as $m_{t}^{e x p}=174 \pm 17 \mathrm{GeV}$ [6], while it was known in the precision analyses before this report that $m_{t}$ needs to be $160-180 \mathrm{GeV}$ to describe all the data in the framework of the electroweak theory.

We surely have come to a position to study "fine structure" of EW (electroweak) corrections as a next step, i.e., to study various parts of them separately which have different properties. In fact, several papers have so far appeared for such a purpose: I examined first whether it is enough for describing $W / Z$-mass data to take into account only the leading-log terms, and found some indication for other-type corrections [7]. Then I proposed an effective procedure to study the non-decoupling top-quark corrections, which are the main part of such "non-leading-log" type contributions [8]. Novikov et al. studied in [2] the Born approximation based on $\alpha\left(M_{Z}\right)$ instead of $\alpha$ (so-called "improved-Born" approximation), and found that it could reproduce all electroweak precision data up to 1993 within the $1 \sigma$ accuracy (see also [3]). Sirlin observed, however, non-Born

effects in low-energy hadron decays [9], while Novikov et al. and I also found some deviation from this approximation in 1994 high-energy data [4, 10]. From a slightly different point of view, Dittmaier et al. [11], and Gambino and Sirlin [12] examined lately the bosonic part of the corrections by using LEP, SLC and Tevatron data, and pointed out their significance.

In this article, I would like to carry out further studies on these subjects using 
the latest data on the weak-boson masses. There is no room for an objection on using $M_{Z}^{\text {exp }}(=91.1888 \pm 0.0044 \mathrm{GeV}$ [13]), while the reason why I focus on the $W$ mass among others is as follows: All the other high-energy precision data are those on $Z$ boson or those at $\sqrt{s} \simeq M_{Z}$. Their accuracy is now reaching the expected highest level. On the contrary, we can expect much more precise determination of $M_{W}$ at Tevatron and LEP II in the near future, although the present $M_{W}^{\exp }$ by $\mathrm{UA} 2+\mathrm{CDF}+\mathrm{D} 0$ has already a fairly good precision. For comparison, the present LEP data on the $Z$-width are combined to give $\Gamma_{Z}^{\text {exp }}=2.4974 \pm 0.0038 \mathrm{GeV}$ 13], i.e., $\pm 0.15 \%$ precision. On the other hand, $M_{W}^{e x p}$ is $80.23 \pm 0.18 \mathrm{GeV}( \pm 0.22 \%$ precision) [14], i.e., already comparable to $\Gamma_{Z}^{\exp }$, and its precision reaches \pm 0.06 $\%$ once $M_{W}$ is measured with an error of $\pm 50 \mathrm{MeV}$ at LEP II [15]. Moreover, the weak-boson mass relation derived from the radiative corrections to $G_{F}$ (the $M_{W}-M_{Z}$ relation) is the freest quantity from various strong-interaction effects, and the meaning of the $W / Z$ masses is of course quite clear Therefore, we can expect very clean tests through the weak-boson mass measurements.

Although the CDF results on the top quark is quite important, its final establishment must come after the confirmation by D0 collaboration. Therefore, I will divide the analyses into two parts:

In $\S 3, m_{t}$-independent analyses are carried out.2 What I study there are the leading-logarithmic approximation, the improved-Born approximation, and the non-decoupling top-quark effects. Testing the last one is particularly important because the existence of such effects is a characteristic feature of theories in which particle masses are produced through spontaneous symmetry breakdown plus large Yukawa couplings.

In $\S 4$, on the other hand, I will study other-type corrections by fully using the

$\sharp 1$ Another popular quantity in electroweak analyses is $\sin ^{2} \theta_{W}$, but it has a number of different definitions in contrast to the weak-boson masses. (Langacker calls this situation "great confusion" in [5].)

$\sharp 2$ This does not mean that the analyses are completely free from the CDF data. I will use the data as a peace of experimental information on $m_{t}$, the meaning of which will become clear at the end of $\S 2$. 
CDF data on $m_{t}$. The EW corrections studied in $\S 3$ are essentially those from fermion loops. Theoretically, however, those from $W, Z$ and the Higgs (i.e., bosonic contributions) are also important. If the top quark is actually very heavy as the CDF data show, we have a good chance to detect the bosonic contribution. This is because the fermionic leading-log terms and the non-decoupling top-quark terms work to cancel each other, and consequently the role of the non-fermionic corrections becomes relatively more significant.

Prior to these two sections, a brief review of the EW corrections to the weakboson masses is given in $\S 2$. The final section is devoted for a conclusion and discussions, where some negative indication to the electroweak theory is pointed out, though it is never serious at present. Since a lot of papers have so far appeared in which comprehensive analyses are performed, as mentioned in the beginning. I wish, therefore, to show here a little different aspect of the precision analyses, which is complementary to those comprehensive analyses.

\section{$\S 2$. EW Corrections to $W / Z$-masses}

I start this section with instructive remarks. Suppose we are trying to test in a theory the existence of some effects phenomenologically. Then, we have to show that the following two conditions are simultaneously satisfied:

- The theory cannot reproduce the data without the terms under consideration, no matter how we vary the remaining free parameters.

- The theory can be consistent with the data by adjusting the free parameters appropriately (i.e., within experimentally and theoretically allowed range), once the corresponding terms are taken into account.

Needless to say, we have to have data and theoretical calculations precise enough to distinguish these two clearly. In those analyses it is safer to be conservative: That is, when we check the first criterion, the smaller number of data we rely on, 
the more certain the result is. On the contrary, for checking the second criterion, it is most trustworthy if we can get a definite conclusion after taking into account all the existing data, preliminary or not.

Now, let us proceed to the main theme. As I mentioned in $\S 1$, the tool of my analyses is the electroweak corrections to the $W / Z$ masses. Through the $O(\alpha)$ corrections to the muon-decay amplitude, these masses are connected as

$$
M_{W}^{2}=\frac{1}{2} M_{Z}^{2}\left\{1+\sqrt{1-\frac{2 \sqrt{2} \pi \alpha}{M_{Z}^{2} G_{F}(1-\Delta r)}}\right\} .
$$

Here $\Delta r$ expresses the corrections, and it consists of several terms with different properties:

$$
\Delta r=\Delta r[\ell . \ell .]+\Delta r\left[m_{t}\right]+\Delta r\left[m_{\phi}\right]+\Delta r[\alpha]
$$

$\Delta r[\ell . \ell$.$] is the leading-log terms from the light charged fermions, \Delta r\left[m_{t}\right]$ and $\Delta r\left[m_{\phi}\right]$ express the non-decoupling top-quark and Higgs-boson effects respectively, and $\Delta r[\alpha]$ is the remaining $O(\alpha)$ non-leading terms. ${ }^{3}$

Equation (2.1) is therefore a formula based on the one-loop calculations (with resummation of the leading-log terms by the replacement $(1+\Delta r) \rightarrow 1 /(1-\Delta r))$. Over the past several years, some corrections beyond the one-loop approximation have been computed to it. They are two-loop top-quark corrections [16] and QCD corrections up to $O\left(\alpha_{\mathrm{QCD}}^{2}\right)$ [17] for $\Delta r\left[m_{t}\right]$. As a result, we have now a formula including $O\left(\alpha \alpha_{\mathrm{QCD}}^{2}\right)$ and $O\left(\alpha^{2} m_{t}^{4}\right)$ effects (see also [18 as reviews). In the following, $M_{W}$ is always computed by incorporating all of these higher-order terms as well, although I will express the whole corrections with these terms also as $\Delta r$ for simplicity.

Let us see here what we can say about the whole radiative corrections as a simple example of applications of the $M_{W}-M_{Z}$ relation and the two criterions I

\footnotetext{
$\sharp 3$ The explicit forms of the first three terms are given in $\S 3$ or $\S 4$, while the last one is too lengthy to show in this article. See, e.g., the Appendix of the second paper in [7].
} 
explained in the beginning. The $W$-mass is computed thereby as

$$
M_{W}^{(0)}=80.941 \pm 0.005 \mathrm{GeV} \text { and } M_{W}=80.33 \pm 0.11 \mathrm{GeV}
$$

for $M_{Z}^{\text {exp }}=91.1888 \pm 0.0044 \mathrm{GeV}$ [13] (and $G_{F}^{e x p}=1.16639 \times 10^{-5} \mathrm{GeV}^{-2}$ ), where $M_{W}^{(0)}$ and $M_{W}$ are those without and with the corrections respectively, and $M_{W}$ is for $m_{t}^{e x p}=174 \pm 17 \mathrm{GeV}$ [6], $m_{\phi}=300 \mathrm{GeV}$ and $\alpha_{\mathrm{QCD}}\left(M_{Z}\right)=0.118$.

As is easily found from Eq. 2.1), $M_{W}^{(0)}$ never depends on $m_{t, \phi}$. So, we can conclude from $M_{W}^{(0)}-M_{W}^{e x p}=0.71 \pm 0.18 \mathrm{GeV}$ and $M_{W}-M_{W}^{e x p}=0.10 \pm 0.21$ GeV that

- $M_{W}^{(0)}$ is in disagreement with $M_{W}^{\text {exp }}$ at more than $3.9 \sigma$ (99.99 \% C.L.),

- $M_{W}$ can be consistent with the data for, e.g., $m_{\phi}=300 \mathrm{GeV}$, which is allowed by the present data $m_{\phi}>61.5 \mathrm{GeV}[19]$.

That is, the two criterions are both clearly satisfied, by which the existence of radiative corrections is confirmed. Radiative corrections were established at $3 \sigma$ level already in the analyses in [20], but where one had to fully use all the available low- and high-energy data. We can now achieve the same accuracy via the weakboson masses alone. Analyses in the following sections are performed in the same way as this, so I do not repeat the explanation on the second criterion below since it is common to all analyses.

Finally, I wish to explain the titles of $\S 3$ and $\S 4$. I mean by " $m_{t}$ independent" that we can test without using $m_{t}^{e x p}$ whether the first criterion is satisfied or not. For the second criterion, we should use all available data to constrain parameter space, as already mentioned.

\section{§3. Top-mass Independent Analyses}

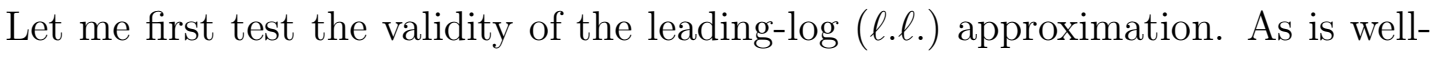
known, leading-log corrections can be easily computed via the one-loop renor- 
malization group equations [21]. It means that any detailed loop calculations are unnecessary if we can describe data within the $\ell . \ell$. approximation.

The explicit form of $\Delta r[\ell . \ell$.$] in Eq.(2.2) is given by$

$$
\Delta r[\ell . \ell .]=-\frac{2 \alpha}{3 \pi} \sum_{f(\neq t)} Q_{f}^{2} \ln \left(\frac{m_{f}}{M_{Z}}\right) \text {, }
$$

where $Q_{f}$ is the electric charge of fermion $f$ in the proton-charge unit, and the sum is both on the flavors and colors except for the top quark. Since $\alpha /(1-\Delta r[\ell . \ell]$. is the QED running coupling at $M_{Z}$ scale within this approximation, I express this combination as $\alpha^{(\ell . \ell .)}\left(M_{Z}\right)$. According to Jegerlehner's computations [22, $\alpha^{(\ell . \ell .)}\left(M_{Z}\right)$ is estimated to be $\alpha^{(\ell . \ell .)}\left(M_{Z}\right)=1 /(127.69 \pm 0.12)$, and thereby the $W$-mass is calculated through

$$
M_{W}^{2}[\ell . \ell .]=\frac{1}{2} M_{Z}^{2}\left\{1+\sqrt{1-\frac{2 \sqrt{2} \pi \alpha^{(\ell . \ell .)}\left(M_{Z}\right)}{M_{Z}^{2} G_{F}}}\right\}
$$

as $M_{W}[\ell . \ell]=.79.798 \pm 0.017 \mathrm{GeV}$. From this and $M_{W}^{e x p}=80.23 \pm 0.18 \mathrm{GeV}$ [14, we get

$$
M_{W}^{e x p}-M_{W}[\ell . \ell .]=0.43 \pm 0.18 \mathrm{GeV}
$$

Therefore, they are in disagreement with each other at about $2.4 \sigma$ (98.4 \% C.L.), which means some non- $\ell . \ell$. corrections are required at this level. The precision level has now become much higher than in my previous analysis [7], where the disagreement level was $1.3 \sigma$ (80.6\% C.L.).

This $\ell . \ell$. approximation can be improved by taking account of some finite terms which are derived by calculating the fermionic one-loop corrections to the photon self-energy explicitly. Indeed, Novikov et al. [2] found that all the precision data up to 1993 are reproduced at $1 \sigma$ level by using

$$
\alpha\left(M_{Z}\right)=\frac{\alpha}{1+\frac{2 \alpha}{3 \pi} \sum_{f(\neq t)} Q_{f}^{2}\left\{\ln \left(\frac{m_{f}}{M_{Z}}\right)+\frac{5}{6}\right\}}
$$


instead of $\alpha^{(\ell . \ell .)}\left(M_{Z}\right)$, where $\alpha\left(M_{Z}\right)$ is known to be $1 /(128.87 \pm 0.12)$ [22]. I examine next whether this "Improved-Born" approximation still works or not. The $W$-mass is calculated within this approximation as

$$
M_{W}[\text { Born }]=79.957 \pm 0.017 \mathrm{GeV}
$$

which leads to

$$
M_{W}^{e x p}-M_{W}[\text { Born }]=0.27 \pm 0.18 \mathrm{GeV}
$$

This means that the Improved-Born approximation is also in disagreement with the data now at $1.5 \sigma$, which corresponds to about $86.6 \%$ C.L.. Although the precision is not yet sufficiently high, it indicates some non-Born terms are needed which give a positive contribution to the $W$-mass. It is noteworthy since the electroweak theory predicts such positive non-Born type corrections unless the Higgs is extremely heavy (beyond TeV scale).

As the final work in this section, I study the non-decoupling top-quark contribution. The significance of testing it was already stressed in $\S 1$. The nondecoupling top contribution to $\Delta r$ is

$$
\begin{aligned}
\Delta r\left[m_{t}\right] & =-\frac{\alpha}{16 \pi s_{W}^{2}}\left\{\frac{3}{s_{W}^{2} M_{Z}^{2}} m_{t}^{2}+4\left(\frac{c_{W}^{2}}{s_{W}^{2}}-\frac{1}{3}-\frac{3 m_{b}^{2}}{s_{W}^{2} M_{Z}^{2}}\right) \ln \left(\frac{m_{t}}{M_{Z}}\right)\right\} \\
& +[\text { higher order corrections }],
\end{aligned}
$$

where $c_{W} \equiv M_{W} / M_{Z}$ and $s_{W}^{2}=1-c_{W}^{2}$. Let me briefly summarize my previous work for this test. What I proposed in [7] is to study what will happen if $\Delta r\left[m_{t}\right]$ would not exist, i.e., to compute the $W$-mass by using the following $\Delta r^{\prime}$ instead of $\Delta r$ in Eq. (2.1):

$$
\Delta r^{\prime} \equiv \Delta r-\Delta r\left[m_{t}\right]
$$

The resultant $W$-mass is denoted as $M_{W}^{\prime}$. The important point is to subtract not only $m_{t}^{2}$ term but also $\ln \left(m_{t} / M_{Z}\right)$ term, though the latter produces only very 
small effects unless $m_{t}$ is extremely large. $\Delta r^{\prime}$ still includes $m_{t}$-dependent terms, but no longer diverges for $m_{t} \rightarrow+\infty$ thanks to this subtraction. I found that $M_{W}^{\prime}$ takes the maximum for the largest $m_{t}$ (i.e., $m_{t} \rightarrow+\infty$ ) and for the smallest $m_{\phi}$ (i.e., $m_{\phi}=61.5 \mathrm{GeV}$ ). The accompanying uncertainty was estimated to be at most $0.03 \mathrm{GeV}$. Therefore,

$$
M_{W}^{\prime}<79.865( \pm 0.030) \mathrm{GeV}
$$

holds for any experimentally allowed values of $m_{t}$ and $m_{\phi}$.

Comparing this inequality with $M_{W}^{e x p}=80.23 \pm 0.18 \mathrm{GeV}$, we have

$$
M_{W}^{e x p}-M_{W}^{\prime}>0.36 \pm 0.18 \mathrm{GeV}
$$

which shows that $M_{W}^{\prime}$ is in disagreement with $M_{W}^{e x p}$ at least at $2 \sigma \quad(=95.5 \%$ C.L.). This means that the electroweak theory is not able to be consistent with $M_{W}^{e x p}$ whatever values $m_{t}$ and $m_{\phi}$ take if the non-decoupling top-quark corrections $\Delta r\left[m_{t}\right]$ would not exist. That is, the latest experimental data of $M_{W, Z}$ demand, independent of $m_{\phi}$, the existence of the non-decoupling top-quark corrections. It is a very important test of the electroweak theory as a renormalizable quantum field theory with spontaneous symmetry breakdown.

\section{$\S 4$. Top-mass Dependent Analyses}

As already mentioned in the Introduction, the existence of the top quark will be established if D0 collaboration also observes events which show its productions. Indeed, it is quite unlikely that the top quark does not exist, apart from how heavy it is. Therefore, I study in this section what we can say when $m_{t}^{e x p}=174 \pm 17$ $\mathrm{GeV}$ is fully used as an input. Concretely, I wish to examine non-fermionic contributions to $\Delta r$ (i.e., the Higgs and gauge-boson contributions within the electroweak theory). It has been pointed out in [11, 12] by using various highenergy data that such bosonic electroweak corrections are now inevitable. For example, Gambino and Sirlin applied the same technique as what I proposed in 
[8] to $\sin ^{2} \theta_{W}$ in $\overline{M S}$ scheme, and found a strong evidence for those effects. I study here whether we can observe a similar evidence in the $M_{W}-M_{Z}$ relation.

According to my procedure, I am to show what occurs if the bosonic contribution would not exist. In this case, in addition to $\Delta r\left[m_{\phi}\right]$ and the bosonic part of $\Delta r[\alpha]$ in Eq.(2.2), the two-loop top-quark corrections have also $m_{\phi}$-dependence, so we have to subtract them as well. I express the resultant $W$-mass calculated for $\Delta r-\Delta r$ [boson] instead of $\Delta r$ as $M_{W}[\mathrm{f}]$ since it receives only the fermionic contribution. $M_{W}[\mathrm{f}]$ is computed for $m_{t}^{e x p}=174 \pm 17 \mathrm{GeV}$ as

$$
M_{W}[\mathrm{f}]=80.44 \pm 0.11 \mathrm{GeV} .
$$

This value is of course independent of the Higgs mass. Equation (4.1) means

$$
M_{W}[\mathrm{f}]-M_{W}^{e x p}=0.21 \pm 0.21 \mathrm{GeV},
$$

which shows that some non-fermionic contribution is necessary at $1 \sigma$ level.

It is of course too early to say from this, e.g., that the bosonic effects were confirmed. Nevertheless, this is an interesting result since we could observe nothing before: For example, the best information on $m_{t}$ before the CDF report was the bound $m_{t}>131 \mathrm{GeV}$ by D0 [23], but we can thereby get only $M_{W}[\mathrm{f}]>80.19$ $( \pm 0.03) \mathrm{GeV}$ (i.e., $\left.M_{W}[\mathrm{f}]-M_{W}^{e x p}>-0.04 \pm 0.18 \mathrm{GeV}\right)$. We will be allowed therefore to conclude that "the bosonic effects are starting to appear in the $M_{W}-M_{Z}$ relation". All the results obtained so far are visually represented in the Figure.

\section{Figure}

For comparison, let us make the same computation for $\Delta M_{W}^{e x p}= \pm 0.05 \mathrm{GeV}$ and $\Delta m_{t}^{e x p}= \pm 5 \mathrm{GeV}$, which will be eventually realized in the future at Tevatron and LEP II. Concretely, $\Delta m_{t}^{e x p}= \pm 5 \mathrm{GeV}$ produces an error of $\pm 0.03 \mathrm{GeV}$ in the $W$-mass calculation. Combining this with the theoretical ambiguity $\Delta M_{W}=$ $\pm 0.03 \mathrm{GeV}$, we can compute $M_{W}[\cdots]-M_{W}^{e x p}$ with an error of about $\pm 0.07 \mathrm{GeV}$. 
Then, $M_{W}[\mathrm{f}]-M_{W}^{e x p}$ becomes $0.21 \pm 0.07 \mathrm{GeV}$ if the central value of $M_{W}^{\exp }$ is the same, by which we can confirm the above statement at $3 \sigma$ level.

We can similar way study the QCD corrections (though it does not match the title of this paper). In this case, I could not find any inequality like Eq.(3.9) which leads to a definite $\left(m_{\phi}\right.$-independent) statement. We can still get, however, some information about the role of the QCD corrections: If we remove these corrections and compute the $W$-mass, we get $M_{W}$ [pure-EW] $=80.48( \pm 0.11) \mathrm{GeV}$ for $m_{\phi}=100 \mathrm{GeV}$. This leads to $M_{W}\left[\right.$ pure-EW] $-M_{W}^{e x p}=0.25 \pm 0.21 \mathrm{GeV}$, which means such a light Higgs is not favored. Indeed, the central value of $M_{W}^{e x p}(=80.23$ $\mathrm{GeV}$ ) becomes to require an extremely heavy Higgs: $m_{\phi}>3 \mathrm{TeV}$. This result tells us that we can no longer neglect QCD effects even in the $M_{W}-M_{Z}$ relation, the least QCD-dependent quantity. Anyway, the electroweak theory is saved by the QCD corrections.

It must be very interesting if we can find moreover the existence of the nondecoupling Higgs effects

$$
\Delta r\left[m_{\phi}\right]=\frac{11 \alpha}{24 \pi s_{W}^{2}} \ln \left(\frac{m_{\phi}}{M_{Z}}\right)
$$

since we still have no phenomenological indication for the Higgs boson. Then, can we in fact perform such a test? It depends on how heavy the Higgs is: If it is much heavier than the weak bosons, then we may be able to test it. If not, however, that test will lose its meaning essentially, since $\Delta r\left[m_{\phi}\right]$ comes from the expansion of terms like $\int_{0}^{1} d x \ln \left\{m_{\phi}^{2}(1-x)+M_{Z}^{2} x-M_{Z}^{2} x(1-x)\right\}$ in powers of $M_{Z} / m_{\phi}$. Here, let us simply assume as an example that we have gained in some way (e.g., at LHC) a bound $m_{\phi}>500 \mathrm{GeV}$. Then, for $\Delta r^{\prime \prime} \equiv \Delta r-\Delta r\left[m_{\phi}\right]$, the $W$-mass (written as $M_{W}^{\prime \prime}$ ) satisfies $M_{W}^{\prime \prime}>80.46 \pm 0.11 \mathrm{GeV}$, where the nondecoupling $m_{\phi}$ terms in the two-loop top-quark corrections were also eliminated. This inequality leads us to $M_{W}^{\prime \prime}-M_{W}^{\exp }>0.23 \pm 0.21 \mathrm{GeV}$.

\footnotetext{
${ }^{\sharp 4}$ Do not take this value too seriously. Perturbation theory is no longer reliable if the Higgs is so heavy [24].
} 
It seems therefore that we may have a chance to get an indirect evidence of the Higgs boson even if future accelerators would fail to discover it. Indeed, the central value of the present $M_{W}^{e x p}$ needs a very heavy Higgs (about $1 \mathrm{TeV}$ [25]: see the next section) though its still large uncertainty allows also a light Higgs. Fortunately or unfortunately, however, the present LEP+SLC data require a light Higgs $(\lesssim 200-300 \mathrm{GeV})[1]$. So, if we get any evidence that the Higgs is heavier than $500 \mathrm{GeV}$, it means that the electroweak theory falls into a trouble. In such a case, it will have little meaning to study various radiative corrections within only the minimal electroweak theory. I will discuss this problem briefly in the next section.

\section{$\S 5$. Conclusion and Discussions}

I have carried out here analyses on (1) $m_{t}$-independent and (2) $m_{t}$-dependent electroweak quantum corrections in the weak-boson mass relation.

In the former part, I tested the leading-log approximation, the ImprovedBorn approximation, and also the non-decoupling top corrections. We could

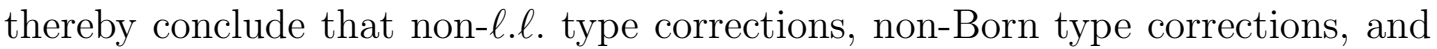
non-decoupling $m_{t}$ contribution are required respectively at about $2.4 \sigma, 1.5 \sigma$, and $2.0 \sigma$ level by the recent data on $M_{W, Z}$. This is a clean, though not yet perfect, test of non-trivial corrections which has the least dependence on hadronic contributions.

Concerning the latter part, we could observe a small indication for nonfermionic contributions (at $1 \sigma$ level, though). This comes from large cancellation between the light-fermion and heavy-top terms, and such contributions can be interpreted as those from the bosonic ( $W / Z$ and the Higgs) corrections. Furthermore, it seemed to be possible to test the non-decoupling Higgs effects if the Higgs boson is heavy (e.g., $\approx 500 \mathrm{GeV}$ ).

On the $m_{t}$-dependent corrections, however, supplementary discussions are nec- 
essary as mentioned in the end of the previous section. In $\S 2$, the $W$-mass with the whole corrections for $m_{t}^{e x p}=174 \pm 17 \mathrm{GeV}$ was computed for $m_{\phi}=300 \mathrm{GeV}$. However, in order for $\left.M_{W}\right|_{m_{t}=174 \mathrm{GeV}}$ to reproduce the central value of $M_{W}^{\exp }(80.23$ $\mathrm{GeV}$ ) within the electroweak theory, the Higgs mass needs to be 1.1-1.2 TeV [25]. Even if we limit discussions to perturbation calculations, such an extremely heavy Higgs will cause several problems [26, 27]. Moreover, the present LEP and SLC data require a light Higgs boson: $m_{\phi} \lesssim 200-300 \mathrm{GeV}$ though at $1 \sigma$ level [1]. This means that we might be caught in a kind of dilemma.

At present, it is never serious since $m_{\phi}$ as low as $60 \mathrm{GeV}$ is also allowed if we take into account $\Delta m_{t}^{e x p}= \pm 17 \mathrm{GeV}$ and $\Delta M_{W}^{e x p}= \pm 0.18 \mathrm{GeV}\left(M_{W}-M_{W}^{e x p}=\right.$ $0.20 \pm 0.21 \mathrm{GeV}$ for $\left.m_{\phi}=60 \mathrm{GeV}\right)$. That is why $\chi^{2}$ takes its minimum at low $m_{\phi}$ even when $M_{W}^{e x p}$ is taken into account in an analysis. However, if $\Delta M_{W}^{e x p}= \pm 0.05$ $\mathrm{GeV}$ and $\Delta m_{t}^{e x p}= \pm 5 \mathrm{GeV}$ are realized as I assumed in $\S 4$, a constraint from the $W$-mass becomes stronger. As an example, let us assume that the central values of $M_{W}^{e x p}$ and $m_{t}^{e x p}$ do not change. Then, $M_{W}-M_{W}^{e x p}$ becomes $0.10 \pm 0.07$ $\mathrm{GeV}$ for $m_{\phi}=300 \mathrm{GeV}$. It means that $m_{\phi}=300 \mathrm{GeV}$ is ruled out at $1.4 \sigma$ level within the minimal standard electroweak theory. Similarly, even $m_{\phi}=400 \mathrm{GeV}$ is not favored though at $1.1 \sigma$ level $\left(M_{W}-M_{W}^{\text {exp }}=0.09 \pm 0.07 \mathrm{GeV}\right)$.

It is of course premature to say thereby, e.g., that we can see some newphysics effects in the very near future. In fact, the above arguments are only an example, and strongly depend on the values of $M_{W}^{e x p}$ and $m_{t}^{e x p}$. The UA2 and CDF data require a little heavier $W$-mass while D0 gives a lighter one as $M_{W}^{e x p}[\mathrm{UA} 2]=80.36 \pm 0.37 \mathrm{GeV}, M_{W}^{e x p}[\mathrm{CDF}]=80.38 \pm 0.23 \mathrm{GeV}$, and $M_{W}^{\exp }[\mathrm{D} 0]=$ $79.86 \pm 0.40 \mathrm{GeV}$ [14]. If a future experimental value converges to a higher value like $M_{W}^{\exp }[\mathrm{UA} 2, \mathrm{CDF}]$, the above problem disappears and we also be able to give the conclusions for $\S 3$ more strongly (but the conclusion for $\S 4$ becomes weaker). On the contrary, if $M_{W}^{e x p}$ moves to $M_{W}^{e x p}[\mathrm{D} 0]$, the situation becomes much more serious. In that case, we will have to consider in earnest new physics which produces opposite contributions to $M_{W}$ and to the other quantities. The top- 
mass measurements can also affect these arguments similarly: If $m_{t}$ moves to a lighter value, the electroweak theory is OK, but if it is found to be heavier, the problem is again serious.

Precise measurements of $M_{W}$ and $m_{t}$ are therefore considerably significant not only for precision tests of the electroweak theory but also for new physics searches beyond this theory.

\section{ACKNOWLEDGEMENTS}

I would like to thank K. Riesselmann and R. Najima for stimulating discussions, S. Errede for sending me useful information on $M_{W}$, J. Fleischer for correspondence on their calculations, and A. J. Buras for reading the manuscript and valuable comments on it. I am also grateful to A. J. Buras and all the members of the particle theory group at TU-München for their warm hospitality, and K. Riesselmann, M. Lautenbacher and S. Herrlich for introducing me to the computer system of this laboratory.

\section{References}

[1] J. Ellis, G. L. Fogli and E. Lisi, Phys. Letters B318 (1993), 148; B333 (1994), 118;

J. Lefrançois, Preprint LAL 93-64 (Talk at the EPS Conference on High Energy Physics, Marseille, France, July 1993);

P. Clarke, Talk at 1994 Rencontres de Moriond, Méribel, France, March 12-19, 1994.

[2] V. A. Novikov, L. B. Okun and M. I. Vysotsky, Mod. Phys. Letters A8 (1993), 2529.

[3] M. Bilenky, K. Kolodziej, M. Kuroda and D. Schildknecht, Phys. Letters B319 (1993), 319; 
G. Altarelli, Preprint CERN-TH.7045/93 (Talk at the EPS Conference on High Energy Physics, Marseille, France, July 1993).

[4] V. A. Novikov, L. B. Okun, A. N. Rozanov and M. I. Vysotsky, Preprint CERN-TH.7217/94 (hep-ph/9404327).

[5] P. Langacker, Preprint UPR-0624-T (hep-ph/9408310);

J. L. Rosner, Preprint EFI 94-38 (hep-ph/9408349).

[6] CDF Collaboration: F. Abe et al., Phys. Rev. Letters 73 (1994), 225; Phys. Rev. D50 (1994), 2966.

[7] Z. Hioki, Phys. Rev. Letters 65 (1990), 683; Zeit. für Phys. C49 (1991), 287.

[8] Z. Hioki, Phys. Rev. D45 (1992), 1814.

[9] A. Sirlin, Phys. Rev. Letters 72 (1994), 1786.

[10] Z. Hioki, Preprint TOKUSHIMA 94-01 (hep-ph/9401340).

[11] S. Dittmaier, D. Schildknecht, K. Kolodziej and M. Kuroda, Nucl. Phys. B426 (1994), 249.

[12] P. Gambino and A. Sirlin, Phys. Rev. Letters 73 (1994), 621.

[13] D. Schaile, Talk at 27th International Conference on High Energy Physics, Glasgow, July 20-27, 1994.

[14] M. Demarteau, H. Frisch, U. Heintz, R. Keup and D. Saltzberg, Joint CDF note/D0 note CDF/PHYS/CDF/PUBLIC/2552 and D0NOTE 2115.

[15] T. Kawamoto, in: Proceedings of the ICEPP Symposium "From LEP to the Planck World", Dec. 17-18, 1992, Univ. of Tokyo, ed. by K. Kawagoe and T. Kobayashi (ICEPP, Univ. of Tokyo, December 1993), p. 55. 
[16] R. Barbieri, M. Beccaria, P. Ciafaloni, G. Curci and A. Viceré, Nucl. Phys. B409 (1993), 105;

J. Fleischer, O. V. Tarasov and F. Jegerlehner, Phys. Letters B319 (1993), 249 ;

M. Consoli, W. Hollik and F. Jegerlehner, Phys. Letters B227 (1989), 167.

[17] F. Halzen and B. A. Kniehl, Nucl. Phys. B353 (1991), 567;

L. Avdeev, J. Fleischer, S. Mikhailov, O. Tarasov, Phys. Letters B336 (1994), 560;

B. A. Kniehl and A. Sirlin, Nucl. Phys. B371 (1992), 141; Phys. Rev. D47 (1993), 883;

B. H. Smith and M. B. Voloshin, Preprint UMN-TH-1241/94 - TPI-MINN94/5-T (hep-ph/9401357).

[18] S. Fanchiotti, B. Kniehl and A. Sirlin, Phys. Rev. D48 (1993), 307;

W. Hollik, Preprint MPI-Ph/93-21 (to appear in Precision tests of the Standard Model, Advanced series on directions in high-energy physics, ed. by Paul Langacker (World Scientific));

B.A. Kniehl, Preprint DESY-93-150 (to appear in Proceedings of International Europhysics Conference on High Energy Physics, Marseille, France, 22-28 July 1993).

[19] T. Kobayashi, Preprint UT-ICEPP 94-05 (Talk at the 22th INS International Symposium on Physics with High Energy Colliders, Tokyo, Japan, March 810, 1994).

[20] U. Amaldi, A. Böhm, L. S. Durkin, P. Langacker, A. K. Mann, W. J. Marciano, A. Sirlin and H. H. Williams, Phys. Rev. D36 (1987), 1385;

G. Costa, J. Ellis, G.L. Fogli, D.V. Nanopoulos and F. Zwirner, Nucl. Phys. B297 (1988), 244. 
[21] F. Antonelli and L. Maiani, Nucl. Phys. B186 (1981), 269;

S. Bellucci, M. Lusignoli and L. Maiani, Nucl. Phys. B189 (1981), 329;

S. Dawson and J. S. Hagelin, Phys.Rev. D23 (1981), 2666.

[22] F. Jegerlehner, in: Proceedings of the 1990 Theoretical Advanced Study Institute in Elementary Particle Physics, ed. by P. Langacker and M. Cvetič (World Scientific, Singapore, 1991), p. 476.

[23] D0 Collaboration: S. Abachi et al., Phys. Rev. Letters 72 (1994), 2138.

[24] J. van der Bij and M. Veltman, Nucl. Phys. B231 (1984), 205;

J. J. van der Bij, Nucl. Phys. B248 (1984), 141.

[25] Z. Hioki and R. Najima, Preprint TOKUSHIMA 94-02 / YCCP-9404 (hepph/9406218).

[26] D. A. Dicus and V. S. Mathur, Phys. Rev. D7 (1973), 3111;

B. W. Lee, C. Quigg and H. Thacker, Phys. Rev. Letters 38 (1977), 883;

Phys. Rev. D16 (1977), 1519;

M. Veltman, Phys. Letters B70 (1977), 253.

[27] L. Durand, B. A. Kniehl and K. Riesselmann, Phys. Rev. Letters 72 (1994), 2534 ;

A. Ghinculov, Preprint Freiburg-THEP 94/08 (hep-ph/9405394). 


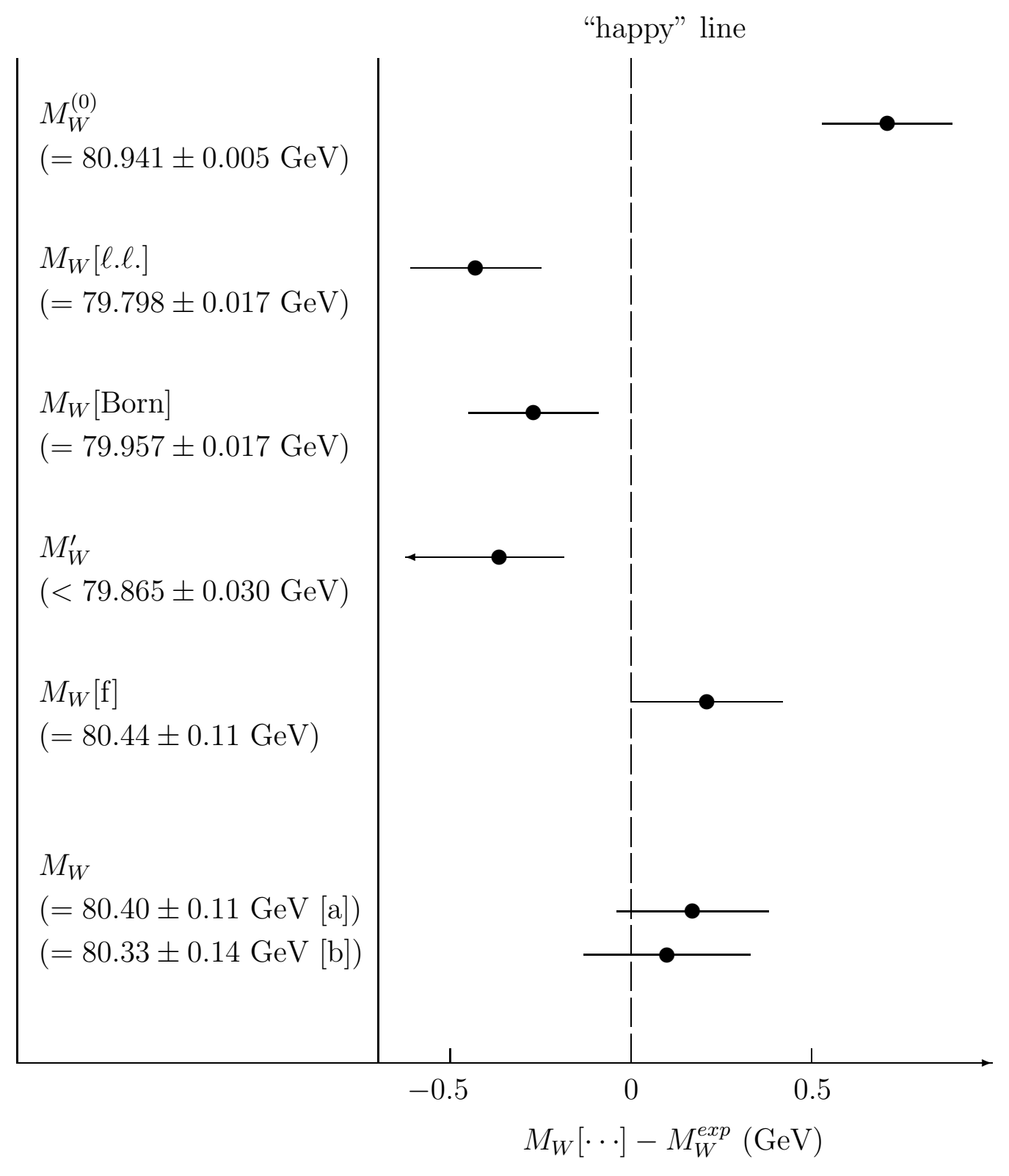

Figure

Deviations of $W$-masses calculated in various approximations from $M_{W}^{e x p}=$ $80.23 \pm 0.18 \mathrm{GeV}$, where $M_{W}[\mathrm{a}, \mathrm{b}]$ are those with the full corrections for $m_{\phi}=100$ $\mathrm{GeV}$ and for $61.5 \mathrm{GeV}<m_{\phi}<1 \mathrm{TeV}$ (the central value is $300 \mathrm{GeV}$ ) respectively. Only $M_{W}[\mathrm{a}, \mathrm{b}]-M_{W}^{e x p}$ cross the "happy" line, and $M_{W}[\mathrm{f}]-M_{W}^{e x p}$ barely touches the line. 